\title{
Hernia research from bench to bed side or "panta rhei in troubled times"
}

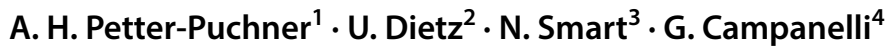

Received: 29 October 2020 / Accepted: 2 November 2020 / Published online: 16 November 2020

๑) Springer-Verlag France SAS, part of Springer Nature 2020

This volume presents some of the most recent and relevant works on experimental and translational hernia research. It cannot be overstated that, despite these extremely troubled and disturbing times, the journal received an amazing variety of valuable contributions, which reflect the complexity and liveliness of our field.

It is an incredible achievement that COVID did not stop the project. We, the managing editors, want to thank the authors, the teams behind them, the reviewers, section editors and everybody at HERNIA investing effort and dedication.

The past decade has shown in multiple ways how experimental work directly influences the routines of surgery and care of hernia patients. This has only been possible because hernia surgeons have always been particularly interested in what was in the pipeline leading from bench to bedside.

Unlike in other surgical fields, the importance of preclinical studies was never really questioned in hernia surgery.

The growing acceptance of atraumatic mesh fixation, leading to the development of highly sophisticated and helpful tools and devices is an example of successful and constructive translation. Today it is beyond any doubt that countless patients have been spared from the once seemingly unavoidable chronic pain by using glues or meshes with micro grips.

Another, more controversial, example is the critical caution exerted today towards biological meshes. First warnings

A. H. Petter-Puchner

alexander.petter-puchner@gesundheitsverbund.at

1 Department of General, Visceral and Oncological Surgery, Klinik Ottakring, Montleartstrasse 37, 1171 Vienna, Austria

2 Department of Surgery, Kantonsspital Olten, Olten, Switzerland

3 Department of Surgery, Royal Devon and Exeter NHS Foundation Trust, Exeter, UK

4 Department of General Surgery, Istituto Clinico Sant'Ambrogio, Milano, Italy on exceeding expectations about this category of implants were phrased on the base of experimental findings. These results were not acclaimed by all and doubts about translational accuracy were raised. Although no unanimous consensus could be reached until today, the discussion and research on all levels have led to an astonishing boost and progress. The sharpened awareness on mesh infection, the definition and classification of contaminated wound fields and the potential use of meshes in such conditions, the development of synthetic absorbable scaffolds (SAS) is a direct result of the passionate debate which has accompanied biologics for many years.

These issues define the current status quo of experimental and clinical challenges in terms of material research and tissue engineering.

Concerning surgical techniques, limits and rationales of open, endoscopic and robotic component separations and the subsequent deposition of a large amount of foreign material are the hot topics.

This topic will be staying with us, whereas, others will even gain more relevance and visibility in the future. In our opinion, new insights on foreign body reaction, silent mesh infection (maybe often not to separate) of polymer meshes, on new tissue and bioengineered products as well as a better understanding of the long term behaviour of SAS will be especially relevant, posing scientific challenges and be game changers in the clinical setting.

We hope you enjoy reading and browsing through the contents of this special volume and that you will be inspired as much as the reviewers and editors were during preparation.

Drs. Campanelli, Petter-Puchner, Smart and Dietz. 


\section{Compliance with ethical standards}

Conflict of interest The authors, Alexander Petter-Puchner, Ulrich Dietz, Neil Smart and Giampiero Campanelli report no interest of conflicts in submitting the editorial.

Ethical approval All procedures performed in studies involving human participants were in accordance with the ethical standards of the institutional research committee and with the 1964 Helsinki declarations and its later amendments or comparable ethical standrds.

Human and animal rights All the procedures performed in the study were in accordance with the ethical standards of the institutional review board/international ethics committee for each center and with the 1964 Helsinki declarations and its later amendments.

Informed consent Informed consent was obtained from all individual participants included in the study.

Publisher's Note Springer Nature remains neutral with regard to jurisdictional claims in published maps and institutional affiliations. 